\title{
Protective Effects of Recombinant Human Erythropoietin against Pressure Overload-Induced Left Ventricular Remodeling and Premature Death in Mice
}

\author{
Wanting Wang, ${ }^{1}$ Yutaka Kagaya, ${ }^{2}$ Yasuhide Asaumi, ${ }^{1}$ Shigefumi Fukui, ${ }^{1}$ \\ Morihiko Takeda ${ }^{1}$ and Hiroaki Shimokawa ${ }^{1}$ \\ ${ }^{1}$ Department of Cardiovascular Medicine, Tohoku University Graduate School of Medicine, Sendai, Japan \\ ${ }^{2}$ Graduate Medical Education Center, Tohoku University Hospital, Sendai, Japan
}

\begin{abstract}
Chronic left ventricular (LV) pressure overload induced by hypertension is one of the most common causes of heart failure. Earlier reports have shown the cardioprotective effects of erythropoietin (EPO). In the present study, we tested the hypothesis that recombinant human EPO exerts a protective effect against pressure-overload induced LV remodeling. Mice subjected to transverse aortic constriction (TAC) $(n=70)$ were randomly assigned to the treatment with phosphate buffer solution (PBS) (TAC-PBS) or EPO (2,000 $\mathrm{U} / \mathrm{kg}$ twice a week) (TAC-EPO). At 8 weeks after TAC, LV weight was comparably increased in both TAC groups compared with sham-operated mice (Sham) (both $P<0.001)$. The treatment with EPO improved the survival of TAC mice as compared with treatment with PBS (80 vs. $47 \%, P<0.01$ ), which was associated with reductions in the extent of myocardial fibrosis and the number of TUNEL positive cardiomyocytes (both $P<0.05$ ). Echocardiography revealed that TAC increased LV chamber diameter and decreased LV fractional shortening compared with Sham $(P<0.05)$, which was ameliorated by the treatment with EPO $(P<0.05)$. In TAC-EPO as compared to TAC-PBS, phosphorylation of STAT3, Akt and eNOS was all increased, while phosphorylation of p38 was decreased (all $P<0.05$ ). Importantly, the expression level of VEGF and the capillary density in LV myocardium were similar among the 3 groups. These results suggest that recombinant human EPO ameliorates the cardiac remodeling and the premature death associated with chronic LV pressure overload through the mechanisms independent of angiogenesis.
\end{abstract}

Keywords: cardioprotection; erythropoietin; left ventricular hypertrophy; pressure-overloaded hearts; ventricular remodeling

Tohoku J. Exp. Med., 2011, 225 (2), 131-143. C 2011 Tohoku University Medical Press

Chronic left ventricular (LV) pressure overload induced by hypertension is one of the most common causes of heart failure (McMurray and Pfeffer 2005). Chronic left ventricular pressure overload elicits LV hypertrophy, an adaptive response to compensate for increased LV wall stress (Panidis et al. 1984). Cardiac remodeling develops when mechanical load and neurohormonal alterations continue (Harada et al. 1998; Iwanaga et al. 1998; Schultz et al. 1999; Schultz et al. 2002), including myocardial interstitial fibrosis, cardiac chamber dilation and cardiac dysfunction, all of which are the critical processes underlying the progression of chronic heart failure (Jessup and Brozena 2003). Although many novel therapeutic tools have been developed for the treatment of heart failure over the past decades, the prognosis of patients with chronic heart failure is still unsatisfactory (Hunt 2005).

Erythropoietin (EPO), which is produced in the adult kidney and fetal liver in mammals, is a cytokine that pro- motes proliferation and terminal differentiation of erythroid progenitor cells by preventing apoptosis (Koury and Bondurant 1990). There is a high degree of sequence homology in the coding region of EPOs among mammalian species. Human and mouse Epos are $76 \%$ identical in nucleotide sequences and $80 \%$ identical in amino acid sequences (Shoemaker and Mitsock 1986; Wen et al. 1993). EPO receptors are expressed not only in hematopoietic lineage cells but also in nonhematopoietic organs (Digicaylioglu et al. 1995; Grimm et al. 2002), including the heart (Calvillo et al. 2003; Parsa et al. 2003; Wright et al. 2004). We have recently reported that endogenous EPOEPO receptor system in nonhematopoietic cells contributes to mobilization of endothelial progenitor cells, their recruitment to the pulmonary artery and suppression of hypoxiainduced pulmonary hypertension in mice (Satoh et al. 2006), and promotes vascular endothelial growth factor (VEGF) expression and angiogenesis in peripheral ischemia

Received August 15, 2011; revision accepted for publication September 6, 2011. doi: 10.1620/tjem.225.131

Correspondence: Yutaka Kagaya, M.D., Ph.D., Graduate Medical Education Center, Tohoku University Hospital, 1-1 Seiryo-machi, Aoba-ku, Sendai, 980-8574 Japan.

e-mail: kagaya@med.tohoku.ac.jp 
in mice (Nakano et al. 2008). We also have demonstrated that endogenous EPO reduces infarct size in patients with acute myocardial infarction subjected to successful primary percutaneous coronary intervention (Namiuchi et al. 2005) and in mice with myocardial ischemia and reperfusion (Tada et al. 2006), and prevents cardiac dysfunction in mice with chronic LV pressure-overload (Asaumi et al. 2007). Recent studies by other laboratories demonstrated that administration of recombinant human EPO elicits cardioprotective effects in animal models of myocardial infarction through JAK-STAT, PI3K-Akt, MAPKs, and endothelial nitric oxide synthase (eNOS) signaling (Smith et al. 2003; Cai and Semenza 2004; Rafiee et al. 2005; Burger et al. 2006; Miyata et al. 2006). However, it is unclear whether exogenous EPO also exerts cardioprotective effects against pressure overload-induced LV remodeling through the mechanisms similar to those of protective effects of endogenous EPO-EPO receptor systems on pressure overload-induced LV remodeling (Asaumi et al. 2007) and those of exogenous EPO in the mouse models of ischemic heart disease (Smith et al. 2003; Cai and Semenza 2004; Rafiee et al. 2005; Burger et al. 2006; Miyata et al. 2006) such as coronary angiogenesis. In the present study, we thus tested the hypothesis that recombinant human EPO ameliorates LV remodeling and survival in mice with chronic LV pressure-overload. We also investigated the mechanisms of cardioprotective effects of exogenous EPO, if any, in such conditions.

\section{Methods}

All the protocols and experimental procedures were approved by the Institutional Committee for the Use and Care of Laboratory Animals of Tohoku University.

\section{Animals}

C57BL/6J male mice, aged 9-11weeks and weighing 22-25g, were used. After baseline body weight, tail-cuff blood pressure and echocardiographic parameters were obtained, transverse aortic constriction (TAC) was performed (Asaumi et al. 2007). Briefly, mice were anesthetized with pentobarbital sodium $(15 \mathrm{mg} / \mathrm{kg}, \mathrm{IP})$ and ventilated with a mouse ventilator (Asaumi et al. 2007). The transverse aorta was constricted via an upper left sternal border with a 7-0 silk surgical thread by ligation of the aorta together with a 27-gauge needle, which was pulled out thereafter (Nishida et al. 2004). Shamoperated mice (Sham) underwent the same procedure but without TAC. The total number of mice used in the present study was 130,9 of them underwent sham operation, and 121 of them were subjected to TAC operation. There were 3 mice $(2 \%)$ died due to excessive bleeding during surgical procedure, and $6 \mathrm{TAC}$ mice $(5 \%)$ died due to acute heart failure within 24 hours after the operation and before randomization to the treatment with PBS or EPO. In the doseresponse experiment, mice with TAC were randomly assigned to the following 4 groups 24 hours after the operation; mice with TAC with the treatment with phosphate buffer solution (PBS) (TAC-PBS) and those with TAC with the treatments with 3 different doses of recombinant human EPO (Chugai, Tokyo, Japan) (500, 2,000, and $4,000 \mathrm{U} / \mathrm{kg})$. PBS or EPO $(500,2,000$, or 4,000 U/kg) was injected subcutaneously twice a week for 8 weeks (Rui et al. 2005; Burger et al. 2006; Li et al. 2006; Miyata et al. 2006) (Fig. 1). Considering the survival, hematocrit levels, and the results of echocardiography in the dose-response experiment, we chose 2,000 U/kg of EPO twice a week (TAC-EPO) in the final study as described in the discussion section.

\section{Hemodynamic measurement and echocardiography}

Systolic blood pressure and heart rate were measured by a noninvasive computerized tail-cuff system (MK-2000, Muromachi Kikai, Tokyo, Japan) at baseline and 2, 4, 6 and 8 weeks after the TAC surgery (Asaumi et al. 2007).

We performed transthoracic echocardiography at baseline and 2, 4, 6 and 8 weeks after the operation using an echocardiographic system equipped with a 12-MHz phased-array transducer (Aplio, Toshiba Medical Systems Corp., Otawara, Japan). Measurements were made offline by an observer who was blinded to the group of each animal. Mice were anesthetized with $2.5 \%$ tribromoethanol $(0.20 \mathrm{mg} / \mathrm{kg}$, IP) because this anesthetic agent is less cardiodepressant than pentobarbital sodium (Asaumi et al. 2007). LV end-diastolic and end-systolic dimensions, LV fractional shortening, interventricular septum thickness at end-diastole and LV posterior wall thickness at end-diastole were measured using M-mode echocardiography, which was assisted by a 2-dimensional LV short axis image at the mid-papillary level (Tada et al. 2006; Asaumi et al. 2007; Fukui et al. 2009). Animals died during echocardiography ( $n=7$ for TAC-EPO) were treated

EPO $500 U / \mathrm{kg}$, EPO 2000U/kg, EPO $4000 \mathrm{U} / \mathrm{kg}$ or PBS twice a week for 8 weeks

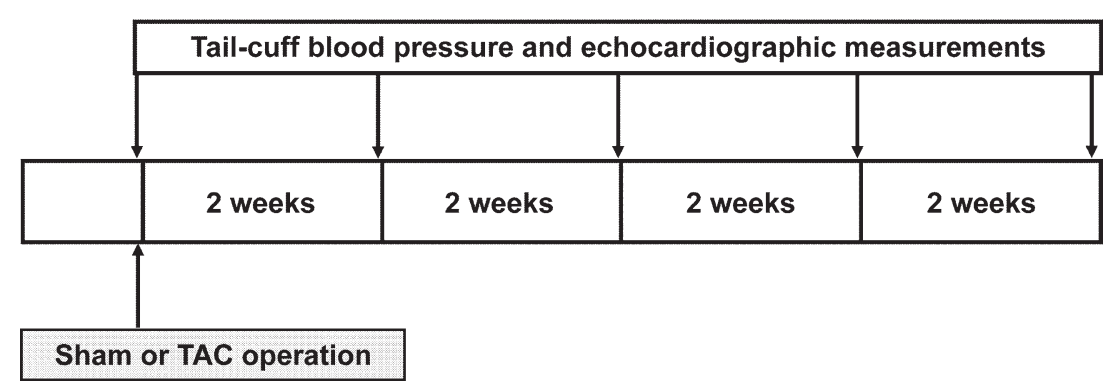

Fig. 1. Experimental protocols. Animals with TAC were randomly divided into groups 24 hours after the operation; mice with TAC injected with phosphate buffer solution (PBS) and those with EPO. 
differently among Figs. 5A, 5B, and $5 \mathrm{C}$ as described in the result section.

\section{Cardiac catheterization}

Cardiac catheterization data were obtained at the end of the 8 -week treatment protocol. Animals were anesthetized with $2.5 \%$ tribromoethanol $(0.38 \mathrm{mg} / \mathrm{kg}$, IP). The right carotid artery was cannulated with a $1.4 \mathrm{~F}$ microtip pressure transducer catheter (Millar Instrument, Houston, TX). After a 2-min period of stabilization, LV peak-systolic pressure (LVSP) and LV end-diastolic pressure (LVEDP) were measured. As indices of contractility and relaxation, the peaks of positive and negative values of the first derivatives of LV pressure $\left(\mathrm{dP} / \mathrm{dt}_{\max }\right.$ and $\left.\mathrm{dP} / \mathrm{dt}_{\min }\right)$ were determined. The cardiac catheterization data were digitized and processed by a computer system (Asaumi et al. 2007). Animals died during cardiac catheterization ( $n=2$ for Sham, $n=4$ for TAC-PBS, and $n=2$ for TAC-EPO) were excluded form the analysis.

\section{Hematocrit and LV weight}

Hematocrit and LV weight were estimated using only animals that survived for 8 weeks after TAC operation. Hematocrit was determined using a hematocrit centrifuge and blood samples collected from the inferior vena cava immediately after laparotomy under anesthesia. The heart was then immediately harvested following thoracotomy and the LV was weighed after the great vessels, atria, and right ventricular free wall were carefully removed. The LV tissue was snap-frozen in liquid nitrogen and stored at $-80^{\circ} \mathrm{C}$ for Western blot analysis or immersed in $10 \%$ buffered formalin or methanol for later histological analyses.

\section{Histological analysis}

Histological analyses were performed using only animals that survived for 8 weeks after surgery. The LV tissue fixed with $10 \%$ buffered formalin was embedded in paraffin, and cut into $3-\mu \mathrm{m}$-thick sections that were perpendicular to the long axis of the LV. The sections were then stained with hematoxylin-eosin, Masson's trichrome or Sirius red. The cross-sectional area of cardiomyocytes was determined using microscopic images of those with both a clear nucleus and intact cellular membrane in hematoxylin-eosin stained sections. At least 30 cardiomyocytes were analyzed per heart. The area of myocardial intersitial fibrosis was determined using Massontrichrome and Sirius red stained sections of LV at the papillary muscle level and was expressed as percent interstitial fibrosis (Li et al. 2006). A computer-aided manipulator program (KS-Analyzer $\mathrm{v} 2.10$, Keyence Corp., Osaka, Japan) was used for those analyses.

For measurement of capillary density, the heart samples immersed in methanol for $24 \mathrm{hrs}$ at $4^{\circ} \mathrm{C}$ were embedded in paraffin. The sections taken perpendicular to the long axis of the LV were immunohistochemically stained with a rat anti-mouse CD31 antibody (M13.3, BD Pharmingen) and then counter-stained with hematoxylin. The number of capillary density was counted in 8-10 random fields in each heart at a magnification of $\times 400$, and each field covered 0.036 $\mathrm{mm}^{2}$. Capillary density was defined as the capillary to cardiomyocyte ratio (Izumiya et al. 2006).

TUNEL assay was performed using an apoptosis detection kit (In situ Apoptosis Detection Kit, TAKARA Bio, Inc., Otsu, Japan) followed by methyl green counterstaining. The number of TUNELpositive cardiomyocytes was counted in 9 random fields in each heart at a magnification of $\times 400$ (Tada et al. 2006).

\section{Western Blot Analysis}

Western blot analysis was performed using only animals that survived for 8 weeks after operation. The frozen LV tissue was homogenized with lysis buffer (pH7.4) containing $25 \mathrm{mM}$ Tris, 150 $\mathrm{mM} \mathrm{NaCl}, 5 \mathrm{mM}$ EDTA, $10 \mathrm{mM}$ sodium pyrophosphate, $10 \mathrm{mM}$ $\beta$-glycerophosphate, $1 \mathrm{mM}$ sodium orthovanadate $\left(\mathrm{Na}_{3} \mathrm{VO}_{4}\right), 1 \%$ (vol/ vol) Triton X-100, 10\% (vol/vol) glycerol, $1 \mathrm{mM}$ dithiothreitol, $1 \mathrm{mM}$ PMSF, and a protease inhibitor cocktail (Sigma, St. Louis, MO). The homogenate was centrifuged at $15,000 \mathrm{rpm}$ for $20 \mathrm{~min}$. The protein concentration of the supernatant was determined with bovine serum albumin as a standard protein. The same amount (20-40 $\mu \mathrm{g}$ for each experiment) of extracted protein was loaded for SDS-polyacrylamide gel electrophoresis and then transferred onto polyvinylidene difluoride membranes. The membranes were probed with antibodies against phospho-ERK (E-4), ERK-1 (K23), JNK (FL), VEGF (147), and actin (I-19) from Santa Cruz Biotechnology Inc., those against phospho-SAPK/JNK (Thr183/Thr185), STAT3, phospho-STAT3 (Tyr705), p38 MAPK, phospho-p38 MAPK (Tyr180/Tyr182), Akt, and phospho-Akt (Ser473) from Cell Signaling Technology, Inc., those against eNOS/NOS Type III and phospho-eNOS (pS1177) from BD Biosciences, Inc. The signals were visualized by the ECL or ECL Plus Western Blotting Detection System (GE Healthcare UK Ltd, Buchinghamshire, UK) (Tada 2006, Asaumi 2007). Image analyzing software (ScnImage) was used for the quantification of protein expression levels.

\section{Statistical analysis}

Data are expressed as mean \pm SEM. Differences among the 3 groups were evaluated using one-way ANOVA followed by Bonferroni correction. Survival was assessed by the standard KaplanMeier analysis with log-rank test. We performed ANOVA with repeated measures followed by Bonferroni's test for comparisons of serial tail-cuff blood pressure, heart rate, and echocardiographic data among the 3 groups. A value of $P<0.05$ was considered to be statistically significant.

\section{Results}

The dose-response experiments to choose an appropriate dose of EPO

Although all the 3 doses of EPO (500, 2,000 and 4,000 $\mathrm{U} / \mathrm{kg}$ twice a week for 8 weeks) significantly and comparably improved survival in mice with TAC as compared with treatment with PBS (Fig. 2A), serial echocardiography revealed a trend favoring 2,000 and 4,000 $\mathrm{U} / \mathrm{kg}$ of EPO as compared with $500 \mathrm{U} / \mathrm{kg}$ of EPO in terms of LV end-diastolic diameter and LV fractional shortening (Fig. 2B). Considering possible harmful effects of the higher hematocrit level achieved by $4,000 \mathrm{U} / \mathrm{kg}$ of EPO compared with 2,000 U/kg of EPO (77\% vs. 69\%) (Fig. 2C), we chose $2,000 \mathrm{U} / \mathrm{kg}$ of EPO, which is comparable to known dosages for cardioprotection, for the final study to investigate whether EPO protects hearts from LV remodeling and premature death in chronic LV pressure overload and the mechanisms of cardioprotective effects of EPO. The results of the final experiments using 2,000 U/kg of EPO twice a week are described in the following sections. 
A

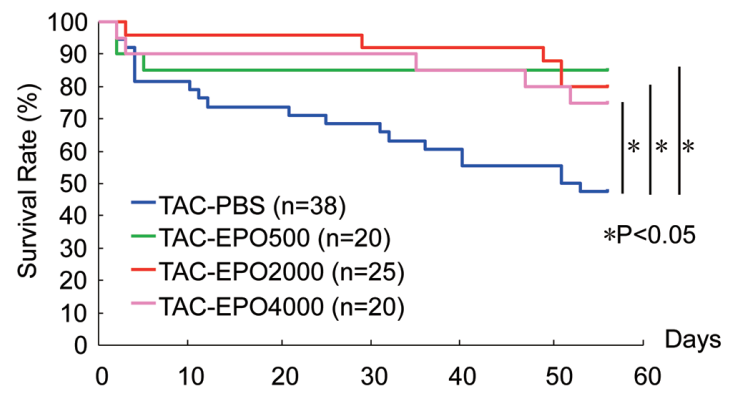

C

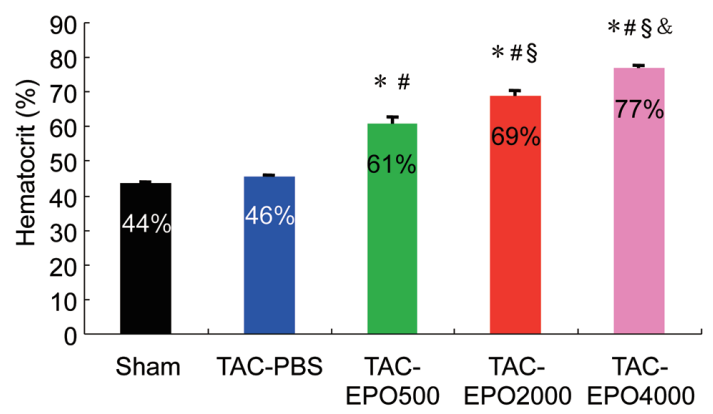

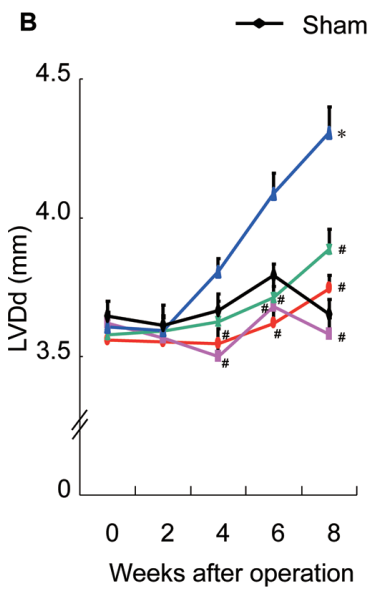
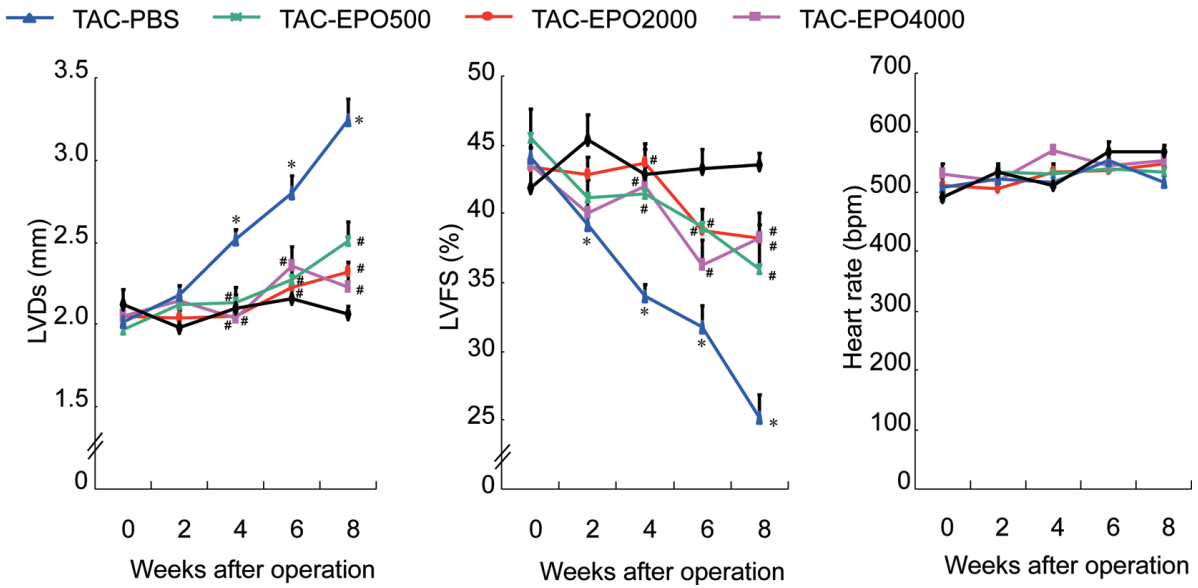

Fig. 2. Survival, echocardiographic data, heart rate, and hematocrit levels in the dose-response experiments.

A. Kaplan-Meier survival curves of TAC-PBS and TAC mice treated with 3 different doses of EPO (500, 2,000 and $4,000 \mathrm{U} / \mathrm{kg}$ twice a week for 8 weeks) (TAC-EPO500, TAC-EPO2000, and TAC-EPO4000, respectively). B. Echocardiographic changes in LV end-diastolic (LVDd) and end-systolic (LVDs) dimensions, LV fractional shortening (LVFS), and heart rate $(n=9$ for Sham; $n=18$ for TAC-PBS; $n=17$ for TAC-EPO500; $n=20$ for TACEPO2000; $n=15$ for TAC-EPO4000). C. Hematocrit levels in Sham, TAC-PBS and TAC mice treated with 3 different doses of EPO $(n=9-20)$. Results are expressed as mean \pm SEM. ${ }^{*} P<0.05$ vs. Sham; $\# P<0.05$ vs. TAC-PBS; $\S P<0.05$ vs. TAC-EPO500; $\& P<0.05$ vs. TAC-EPO2000.

Effects of 8-week treatment with EPO on hemodynamics, hematocrit and $L V$ weight after TAC

Tail-cuff blood pressure and heart rate were comparable among Sham, TAC-PBS and TAC-EPO during the 8-week treatment period after TAC (Fig. 3). As shown in Table $1, \mathrm{LV}$ weight/body weight ratio was significantly increased in both TAC-PBS and TAC-EPO as compared with Sham $(P<0.001$ for each), although there was no significant difference in the extent of increases in LV weight between TAC-PBS and TAC-EPO. Hematocrit at the end of the 8-week treatment protocol was significantly increased in TAC-EPO as compared with Sham and TAC-PBS $(P<$ 0.001 for each).

\section{Effects of EPO on interstitial fibrosis and size of cardiomy- ocytes}

Representative histological micrographs of the LV of the 3 groups at 8 weeks after operation are shown in Fig. 4A. The extent of LV interstitial fibrosis determined by staining with Masson's trichrome and Sirius red was significantly increased in TAC-PBS as compared with Sham. The
EPO treatment for 8 weeks significantly reduced the extent of myocardial interstitial fibrosis (Fig. 4B). TAC significantly and comparably increased the cross-sectional area of cardiomyocytes regardless of treatment with PBS or EPO (Fig. 4A and 4B).

\section{Survival over 8 weeks after TAC operation}

Cumulative survival for 8 weeks after the induction of TAC was significantly improved in TAC-EPO as compared with TAC-PBS ( $80 \%$ vs. $47 \%, P<0.01$; Fig. 5A). Postmortem examination revealed severe pulmonary congestion in most of the mice that died within 8 weeks after TAC regardless of treatment with PBS or EPO (data not shown). We excluded 7 TAC-EPO mice that had died during echocardiography from the analysis of survival in Fig. 5A. When we treated those mice as ones that had dropped out from the study, the survival rate was $84 \%$ for TAC-EPO, and was significantly higher than that of TACPBS (47\%, $P<0.05$; Fig. 5B). Death during echocardiography under anesthesia is not natural death. However, when we treated the death as one equal to natural death due to LV 

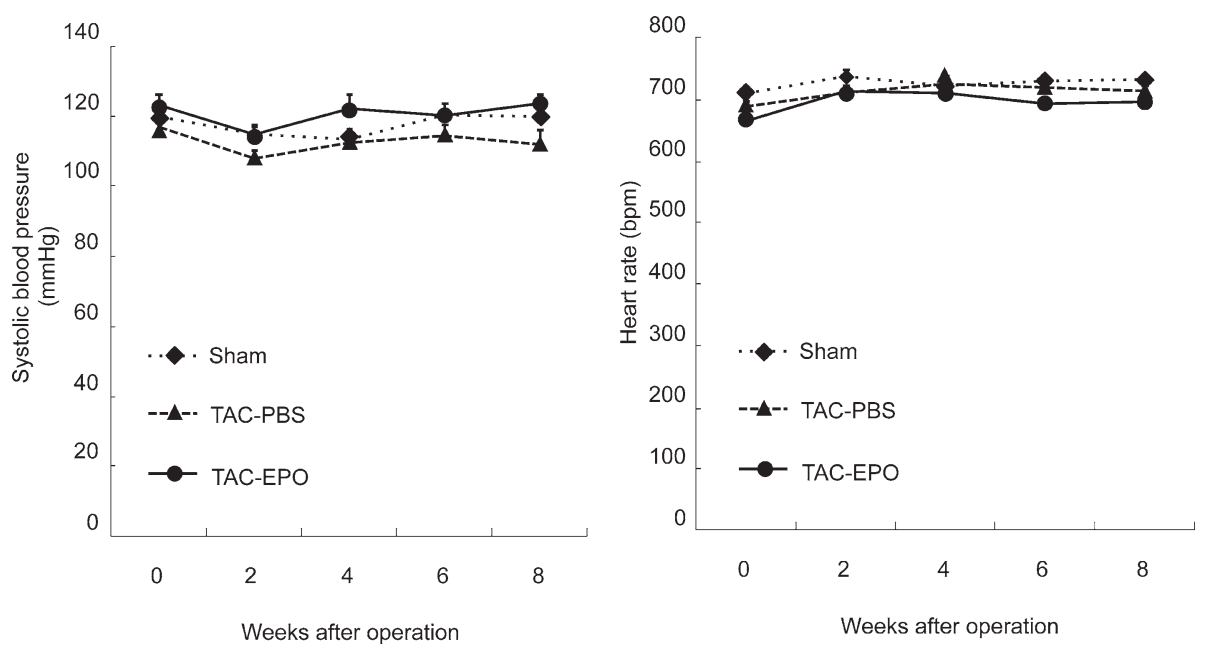

Fig. 3. Tail-cuff systolic blood pressure and heart rate. Results are expressed as mean \pm SEM. $n=9$ for Sham; $n=18$ for TAC-PBS; $n=20$ for TAC-EPO.

Table 1. Characteristics of mice at 8 weeks after operation.

\begin{tabular}{lccc}
\hline & $\begin{array}{c}\text { Sham } \\
(n=9)\end{array}$ & $\begin{array}{c}\text { TAC-PBS } \\
(n=18)\end{array}$ & $\begin{array}{c}\text { TAC-EPO } \\
(n=20)\end{array}$ \\
\hline Body weight, g & $25.5 \pm 0.7$ & $26.0 \pm 0.4$ & $25.6 \pm 0.3$ \\
LV weight, mg & $78.4 \pm 3.2$ & $142.7 \pm 6.1^{*}$ & $136.78 \pm 3.5^{*}$ \\
LV weight/body weight ratio & $3.07 \pm 0.07$ & $5.47 \pm 0.18^{\S}$ & $5.34 \pm 0.11^{\S}$ \\
Hematocrit, \% & $43.6 \pm 0.7$ & $45.6 \pm 0.5$ & $68.8 \pm 1.6^{\S,}$ \\
\hline
\end{tabular}

Results are expressed as mean \pm SEM. $* P<0.05$ vs. Sham; $\$ P<0.001$ vs. Sham; $₫ P<0.001$ vs. TAC-PBS.

pressure overload, the survival rate was $63 \%$ for TAC-EPO, and still tended to be higher than that of TAC-PBS $(47 \%, P$ $=0.086$; Fig. 5C).

\section{Effects of EPO on LV dysfunction induced by TAC}

Representative echocardiographic tracings in the 3 groups are demonstrated in Fig. 6A, and serial echocardiographic parameters in the 3 groups during the 8-week treatment period are shown in Fig. 6B-G. In TAC-PBS, LV pressure overload resulted in significant enlargements of LV end-diastolic and end-systolic diameters and a deterioration of LV fractional shortening, all of which progressed over the course of 8 weeks. These alterations in echocardiographic parameters were significantly ameliorated in TACEPO as compared with TAC-PBS (all $P<0.05$ ). The interventricular septum thickness at end-diastole was significantly increased in TAC-PBS as compared with Sham $(P<0.05)$, and it was further thickened in TAC-EPO as compared with TAC-PBS $(P<0.05)$.

The results of cardiac catheterization performed at the end of the 8-week treatment protocol are shown in Table 2. LV peak-systolic pressure was significantly increased in TAC-PBS as compared with Sham $(P<0.05)$. LV peaksystolic pressure in TAC-EPO was significantly higher than that in TAC-PBS $(P<0.05)$. Both $\mathrm{LV} \mathrm{dP/dt}$ max and $\mathrm{LV} \mathrm{dP/}$ $\mathrm{dt}_{\min }$ were significantly increased in TAC-EPO as compared with TAC-PBS. LV end-diastolic pressure tended to be increased in both TAC-PBS and TAC-EPO as compared with Sham, although the differences did not reach statistical significance.

Effects of EPO on the phosphorylation of signaling proteins

The Epo treatment for 8 weeks after TAC significantly increased the protein expression ratio of phospho-STAT3/ STAT3 in the LV myocardium of TAC mice as compared with Sham and TAC-PBS (Fig. 7A and 7B). The ratio of phospho-Akt/Akt in the LV myocardium of TAC-EPO was also significantly higher than those of Sham and TAC-PBS (Fig. 7A and 7B). Although the ratio of phospho-p38/p38 was significantly decreased in TAC-EPO as compared with TAC-PBS (Fig. 7A and 7B), the ratios of phospho-ERK1/2 / ERK1/2 and phospho JNK/JNK did not differ significantly between TAC-PBS and TAC-EPO (Fig. 7A and 7B). Protein expression levels of phosphorylated eNOS normalized by that of $\beta$-actin in LV myocardium was significantly increased in TAC-EPO as compared with TAC-PBS (Fig. 7A and 7C). 
A
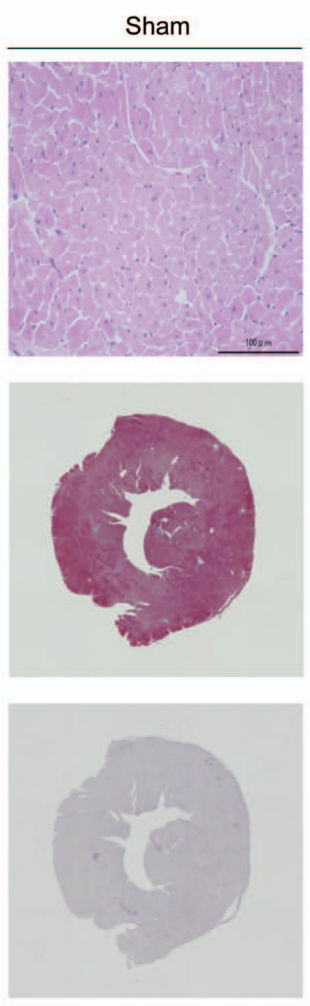

TAC-PBS
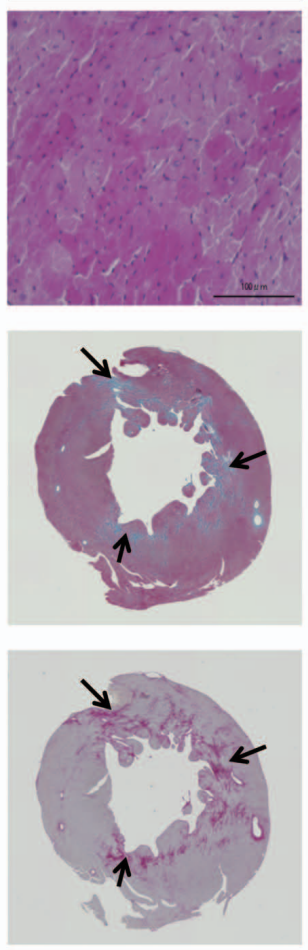

TAC-EPO
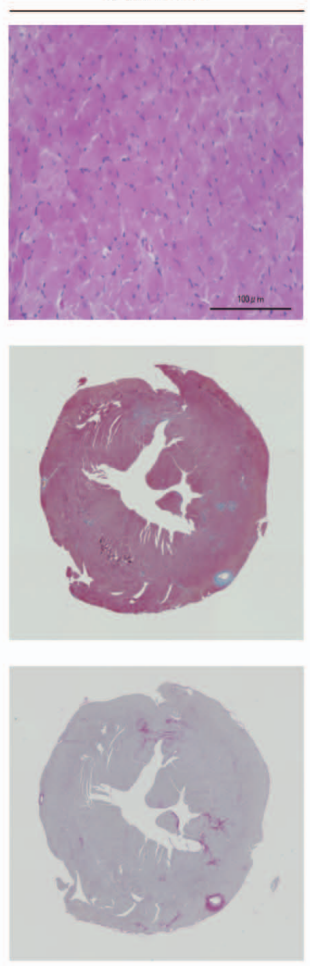

B
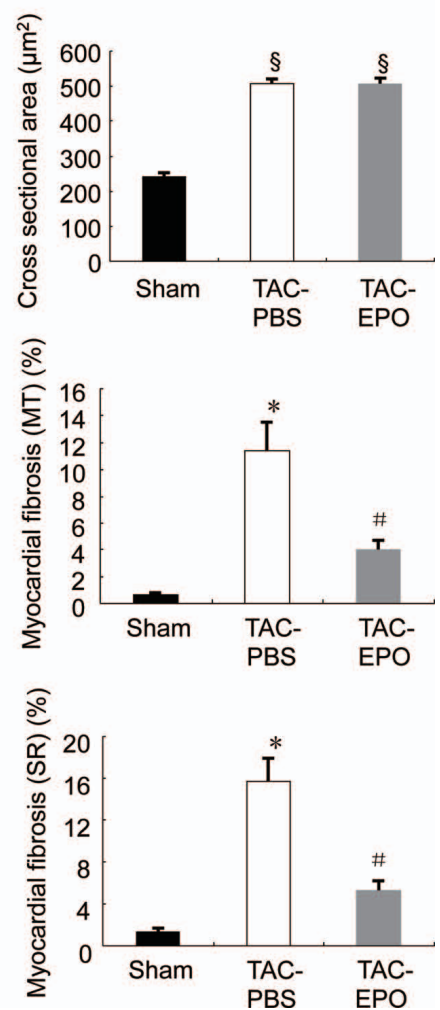

Fig. 4. Histological analysis.

A. Representative histological micrographs of LV myocardium stained with hematoxylin-eosin (top), Masson's trichrome (middle), and Sirius red (bottom) at 8 weeks after sham operation or TAC. The arrowheads indicate massive myocardial fibrosis in TAC-PBS. B. Quantitative analyses of the cross-sectional area of cardiomyocytes (top) and the fibrosis area of LV myocardium stained with Masson's trichrome (MT, middle) and Sirius red (SR, bottom) $(n=4$ to 6$)$. Results are expressed as mean \pm SEM. ${ }^{*} P<0.05$ vs. Sham; $\# P<0.05$ vs. TAC-PBS; $\S P<0.001$ vs. Sham.
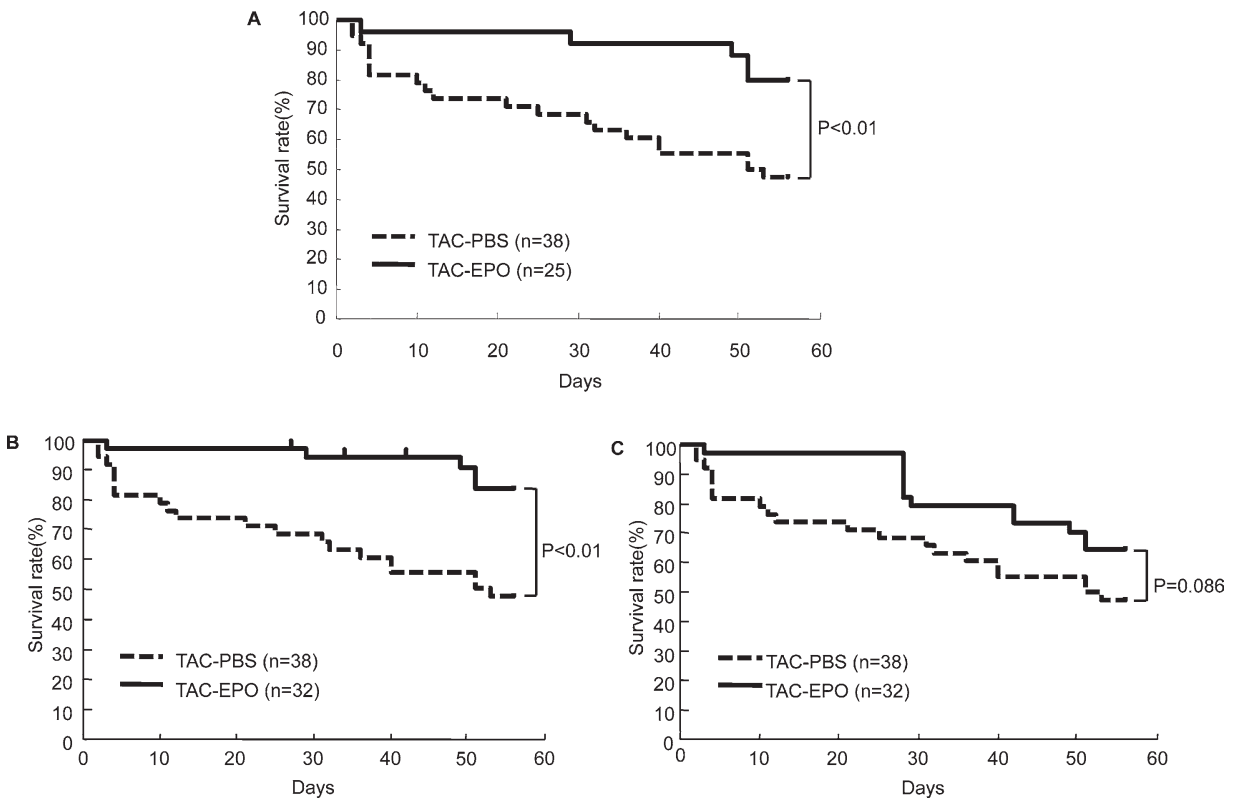

Fig. 5. Kaplan-Meier survival curves.

A. TAC-EPO mice that had died during echocardiography $(n=7)$ were excluded form the analysis.

B. TAC-EPO mice that had died during echocardiography $(n=7)$ were treated as ones that had dropped out from the study.

C. Death during echocardiography ( $n=7$ for TAC-EPO) was treated as one equal to natural death due to LV pressure overload. 
A
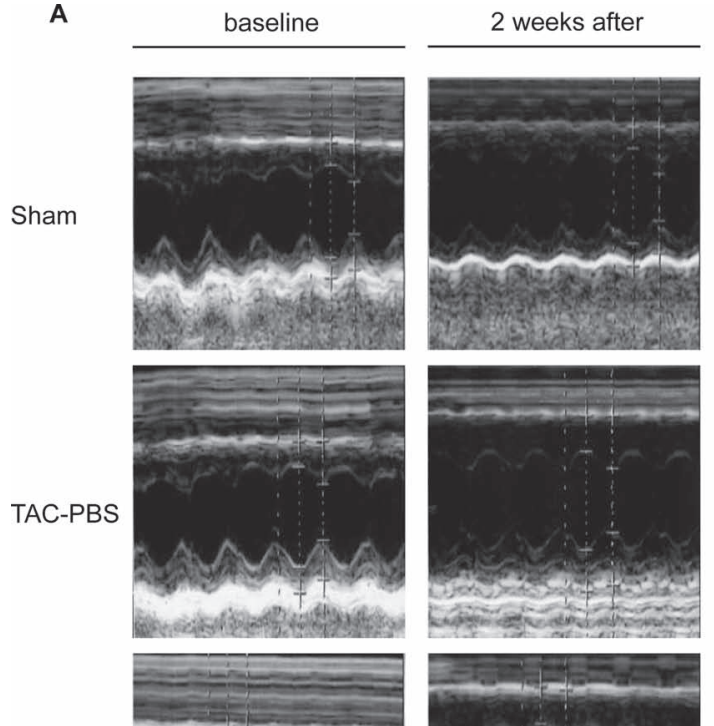

TAC-EPO
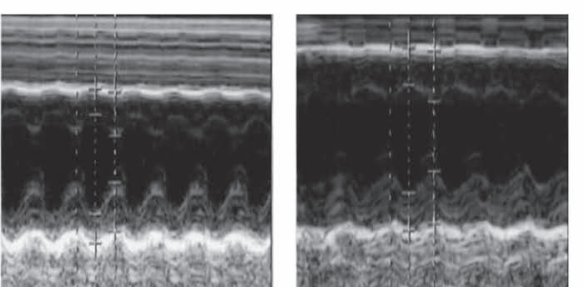

4 weeks after
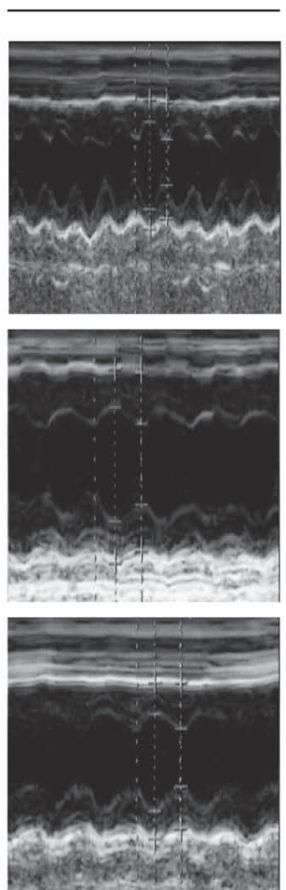

6 weeks after
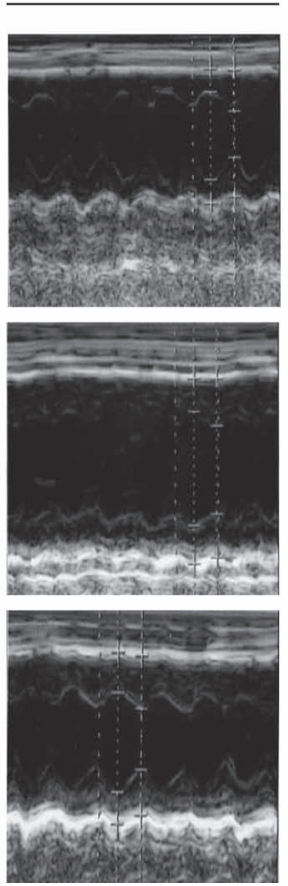

8 weeks after
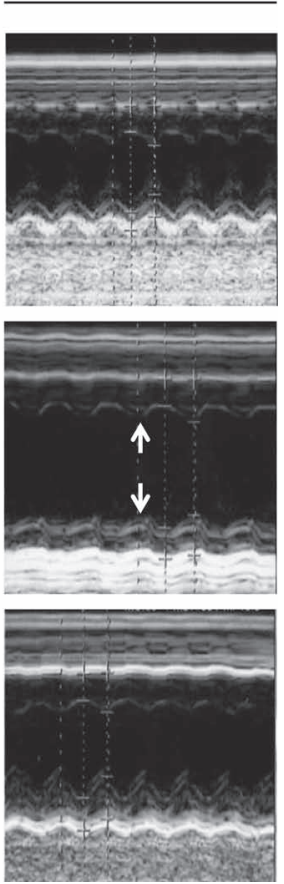

B

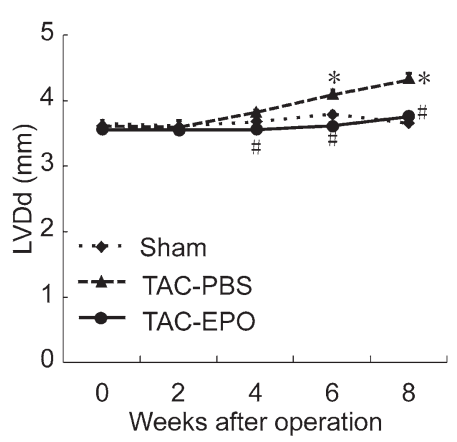

E

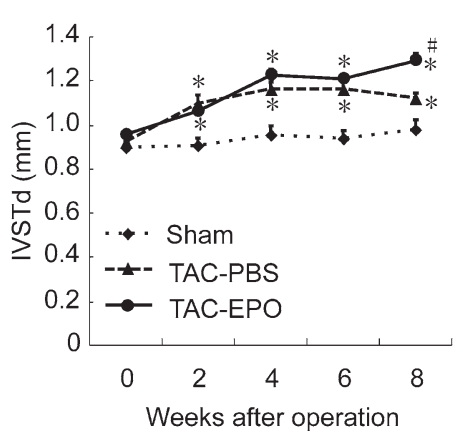

C

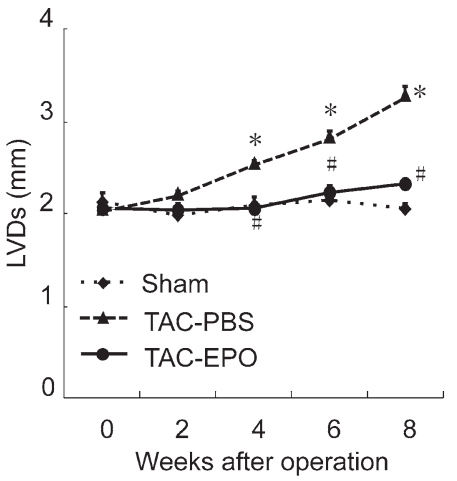

$\mathbf{F}$

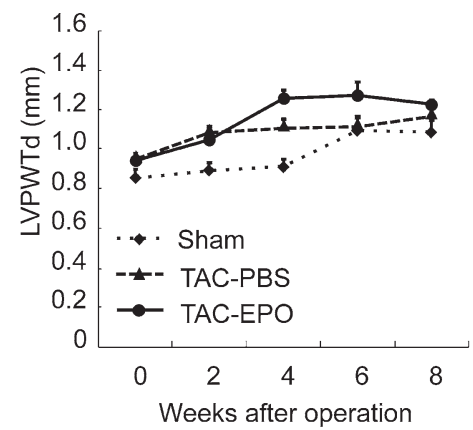

D

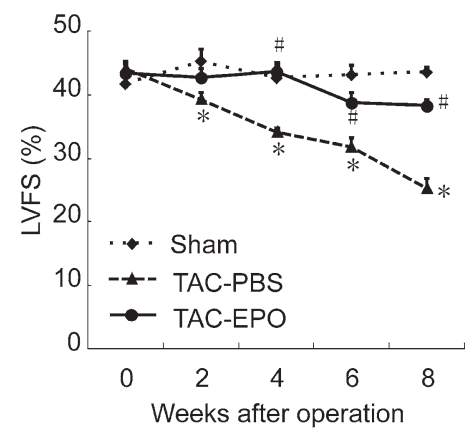

G

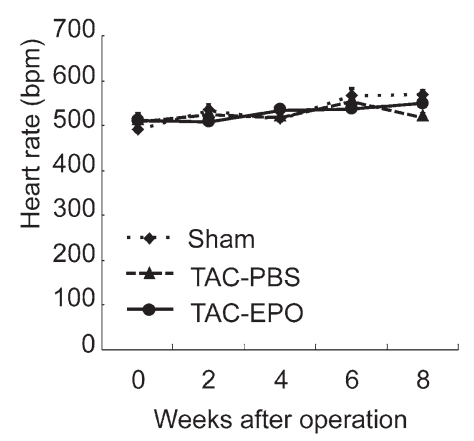

Fig. 6. Serial echocardiographic data.

A. Representative transthoracic M-mode echocardiographic tracings from Sham, TAC-PBS, and TAC-EPO before and at 2, 4, 6, and 8 weeks after operation. The arrowheads indicate severely impaired LV wall motion in TAC-PBS.

B-G. Echocardiographic changes in LV end-diastolic (LVDd) and end-systolic (LVDs) dimensions, LV fractional shortening (LVFS), interventricular septum thickness at end-diastole (IVSTd), and LV posterior wall thickness at enddiastole (LVPWTd), and heart rate ( $n=9$ for Sham; $n=18$ for TAC-PBS; $n=20$ for TAC-EPO). Results are expressed as mean \pm SEM. $* P<0.05$ vs. Sham; $\# P<0.05$ vs. TAC-PBS. 
Table 2. Results of cardiac catheterization at 8 weeks after operation.

\begin{tabular}{lccc}
\hline & $\begin{array}{c}\text { Sham } \\
(n=7)\end{array}$ & $\begin{array}{c}\text { TAC-PBS } \\
(n=14)\end{array}$ & $\begin{array}{c}\text { TAC-EPO } \\
(n=18)\end{array}$ \\
\hline LV systolic pressure, mmHg & $86.4 \pm 3.2$ & $135.7 \pm 6.5 *$ & $161.5 \pm 4.2 *, \#$ \\
LV end-diastolic pressure, mmHg & $3.0 \pm 0.6$ & $7.5 \pm 1.5$ & $6.9 \pm 1.1$ \\
LV dp/dt max, mmHg/sec & $6,110 \pm 570$ & $5,640 \pm 270$ & $6,540 \pm 240^{\#}$ \\
LV -dp/dt min, mmHg/sec & $7,300 \pm 640$ & $6,490 \pm 400$ & $8,590 \pm 450^{\#}$ \\
Heart rate (catheterization), bpm & $483 \pm 25$ & $452 \pm 13$ & $468 \pm 12$ \\
\hline
\end{tabular}

Results are expressed as mean \pm SEM. $* P<0.05$ vs. Sham; $\# P<0.05$ vs. TAC-PBS. LV dp/dt max, maximum first time derivative of LV pressure; and LV -dp/dt min, minimum first time derivative of LV pressure.

A

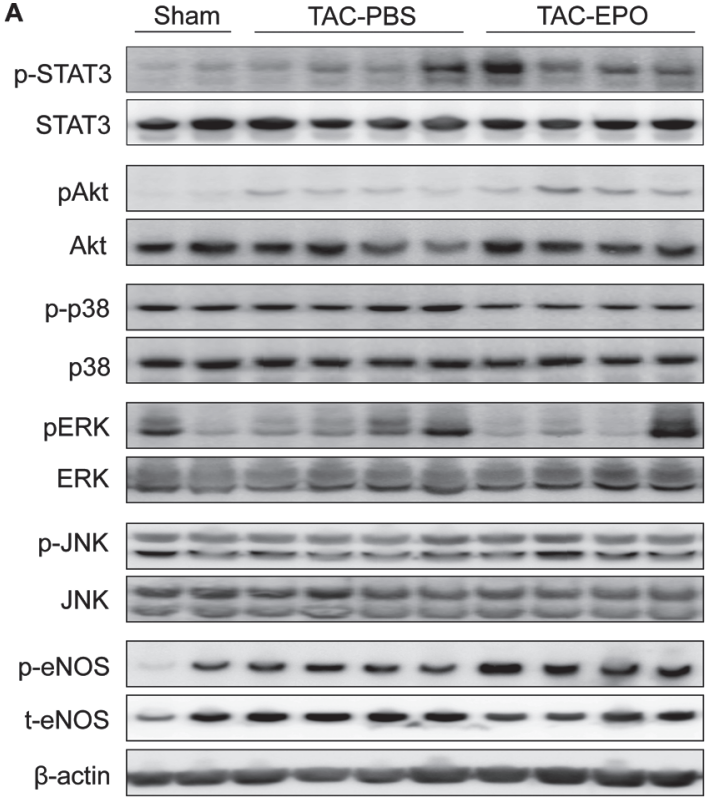

C

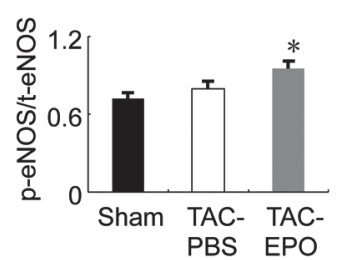

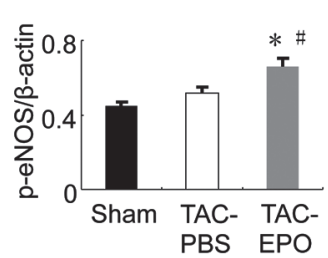

B
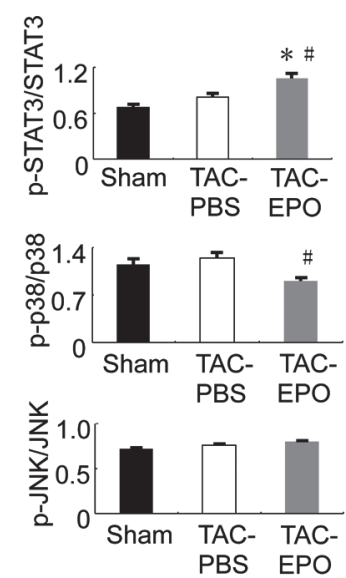

D

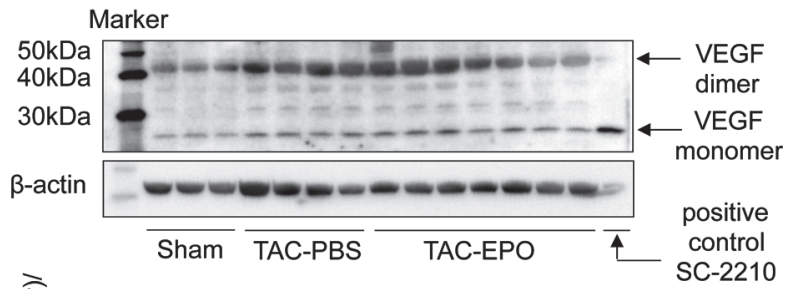

E

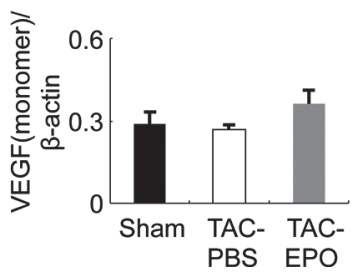

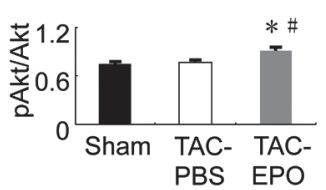

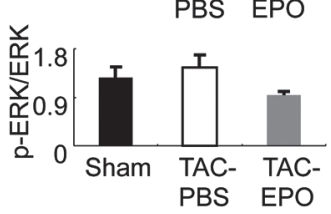

PBS EPO

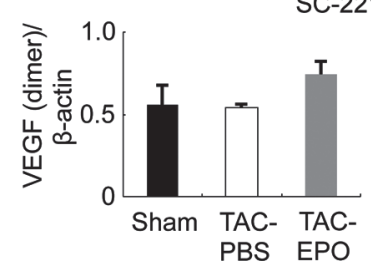

Fig. 7. Representative Western blots and the results of quantitative analysis of LV myocardial tissue at 8 weeks after operations.

A, D. Representative Western blots. B, C, E. Results of quantitative analysis. Results are expressed as mean $\pm \mathrm{SEM}(n$ $=5-9) . * P<0.05$ vs. Sham; $\# P<0.05$ vs. TAC-PBS.

No effects of EPO on VEGF expression or myocardial capillary density

Protein expression levels of VEGF in LV myocardium normalized by that of $\beta$-actin at 8 weeks after sham or TAC operation did not differ significantly among Sham, TACPBS, and TAC-EPO (Fig. 7D and 7E). Immunohistochemical analysis revealed that there was no significant difference in myocardial capillary density among the 3 groups (Fig. 8).
Effects of EPO on apoptosis of cardiomyocytes after TAC

Although the percentage of TUNEL positive cells in LV myocardium was small in all the 3 groups, it was significantly increased in TAC-PBS as compared with Sham $(P<$ 0.05) (Fig. 9). The EPO treatment for 8 weeks significantly reduced the percentage of TUNEL positive cells $(P<0.05)$.

\section{Discussion}

The novel finding of the present study was that treatment with recombinant human EPO ameliorates cardiac remodeling and improves survival in mice with 

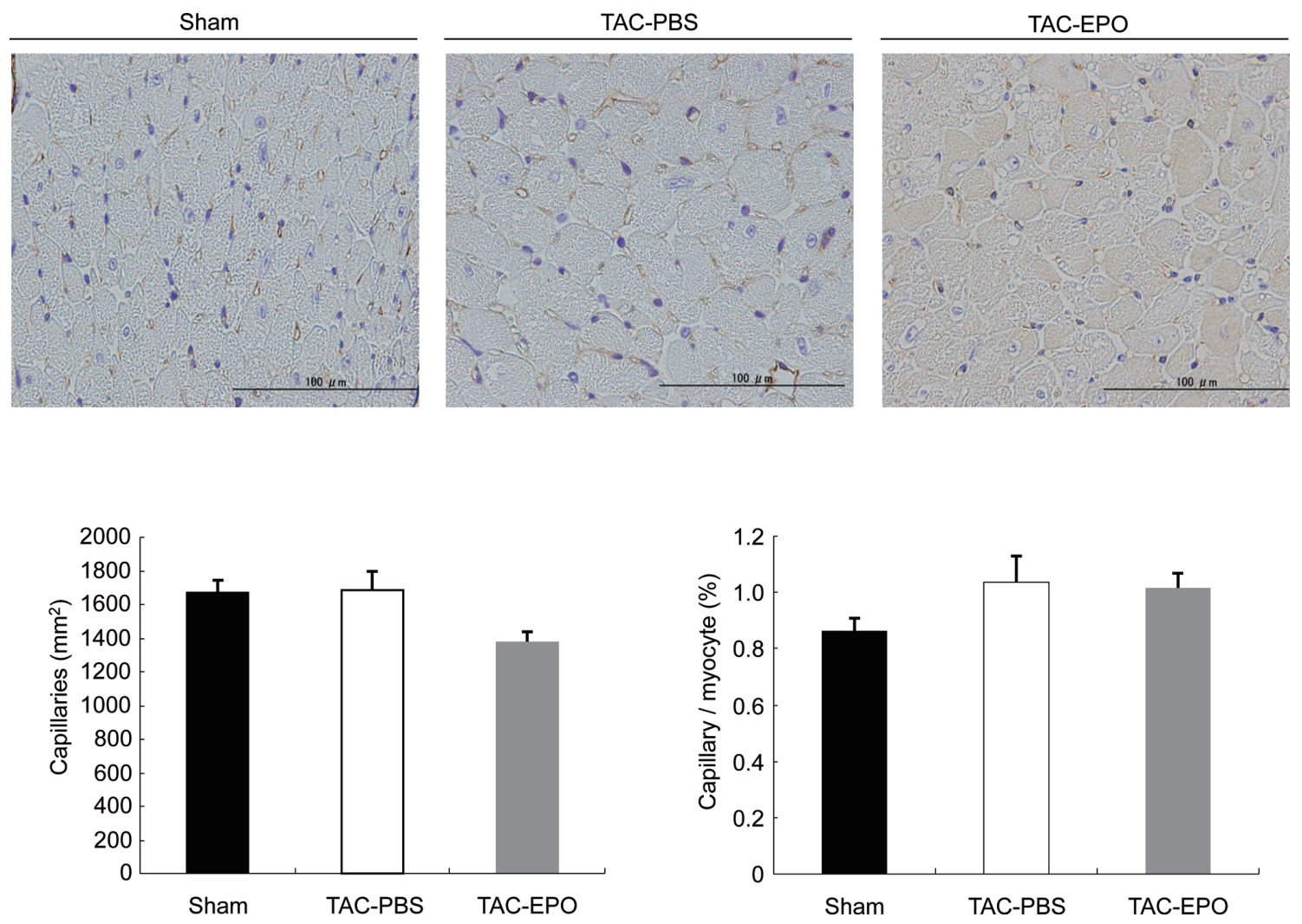

Fig. 8. Representative CD31 immunostaining images and numbers of CD31-positive capillaries in LV myocardium at 8 week after operations $(n=5-9)$. Results are expressed as mean \pm SEM.

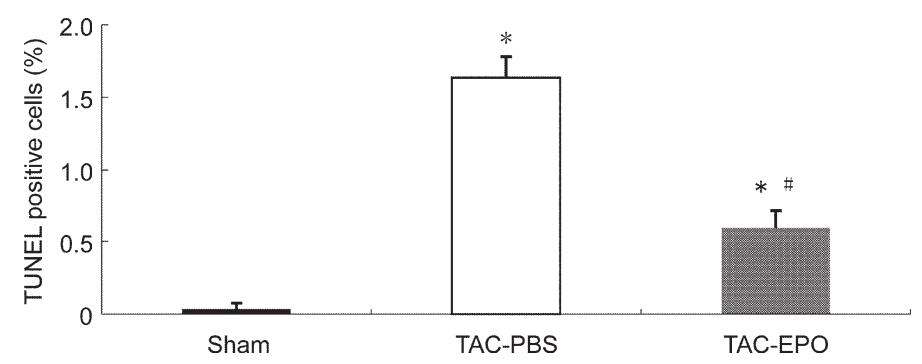

Fig. 9. Numbers of TUNEL staining-positive cardiomyocytes in LV myocardium at 8 weeks after operations $(n=4-6)$. Results are expressed as mean \pm SEM. ${ }^{*} P<0.05$ vs. Sham; $\# P<0.05$ vs. TAC-PBS.

chronic LV pressure overload. The reduced susceptibility to cardiac remodeling and premature death in TAC mice treated with EPO was associated with accelerated phosphorylation of STAT3, Akt, and eNOS, attenuated phosphorylation of $\mathrm{p} 38$, and a reduction in the number of apoptotic cardiomyocytes. In contrast to the previous studies by others using animal models of myocardial infarction (van der Meer et al. 2005; Nishiya et al. 2006; Ueda et al. 2010), the expression of VEGF and capillary density in LV myocardium did not change significantly by the treatment with EPO in our murine model of LV pressure overload. These results suggest that recombinant human EPO ameliorates cardiac remodeling and improves survival in pressure-overloaded hearts, which could be explained at least in part by the alteration of phosphorylation state of several key molecules located in the multiple downstream signaling pathways of EPO receptors, but not by coronary angiogenesis (Fig. 10).

Protective effects of EPO against $L V$ remodeling and premature death induced by chronic $L V$ pressure overload

We demonstrated for the first time that recombinant human EPO exerts protective effects against LV remodeling and premature death induced by chronic LV pressure overload in mice. Serial echocardiography demonstrated that the EPO treatment for 8 weeks after TAC significantly ameliorated enlarged LV chamber dimension and reduced LV fractional shortening. The EPO treatment also significantly reduced myocardial interstitial fibrosis, cardiomyocyte apoptosis and improved survival. The 


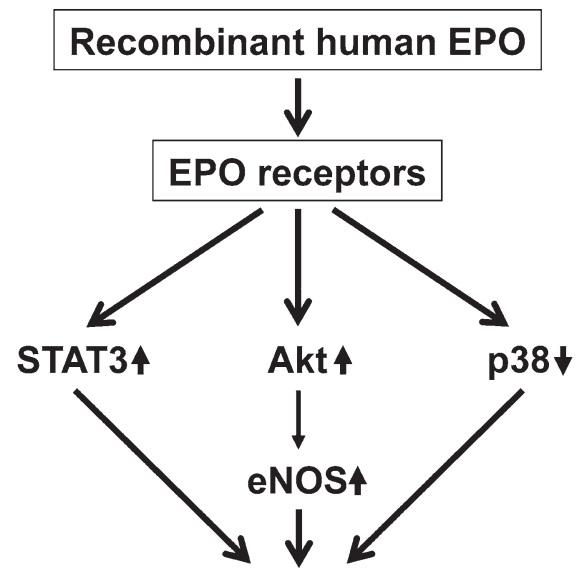

LV systolic and diastolic performance 4 Myocardial interstitial fibrosis $\downarrow$ Cardiomyocyte apoptosis $\downarrow$

\section{Cardiac remodeling $\downarrow$ Premature death $\downarrow$}

Fig. 10. Summary of the present study on the possible mechanisms for the protective effects of recombinant human EPO on pressure-overloaded hearts.

The Epo-Epo receptor system mediates accelerated phosphorylation of STAT3, Akt and eNOS, and depressed phosphorylation of $\mathrm{p} 38$, resulting in the amelioration of cardiac remodeling and the prevention of premature death.

present findings are consistent with those of previous studies by others, in which recombinant human EPO ameliorated LV remodeling in rodent animals with myocardial infarction (Nian et al. 2004; Ueda et al. 2010) and in doxorubicin-induced cardiomyopathy ( $\mathrm{Li}$ et al. 2006). We have previously reported that LV remodeling and survival one week after TAC were significantly deteriorated in mice deficient in EPO receptors exclusively in nonhematopoietic cells as compared with wild-type mice with TAC (Asaumi et al. 2007). This finding suggests that endogenous EPO-EPO receptor system in nonhematopoietic cells exerts important protective effects against cardiac remodeling and premature death induced by LV pressure overload. The present findings suggest that exogenous EPO-mediated signaling elicits further beneficial effects on both cardiac remodeling and survival in pressureoverloaded hearts in addition to the favorable effects already brought by endogenous EPO-EPO receptor system in nonhematopoietic cells.

The present finding is partially inconsistent with those of previous studies by others using a myocardial infarction model (van der Meer et al. 2005; Nishiya 2006; Ueda et al. 2010) and our previous study (Asaumi et al. 2007), as we did not observe an increase in the expression of VEGF or capillary density in LV myocardium in response to the treatment with EPO in the present study. The differences in the models used (LV pressure overload vs. myocardial infarction) and the source of EPO (exogenous EPO vs. endogenous EPO) might explain the inconsistent results. Indeed, Li et al. (2006) reported that recombinant human EPO exerted a cardioprotective effects in mice with doxorubicin-induced cardiomyopathy without affecting coronary angiogenesis.

Possible molecular mechanisms for the protective effects of recombinant human EPO against pressure overloadinduced $L V$ remodeling

Several possible mechanisms may be involved in the EPO-induced amelioration of cardiac remodeling and improved survival after TAC. Recombinant human EPO has been shown to exert protective effects against myocardial injury in animal models of acute myocardial infarction (van der Meer et al. 2005; Nishiya et al. 2006; Ueda et al. 2010), post-infarction LV remodeling (Nian et al. 2004), and doxorubicin-induced cardiomyopathy (Li et al. 2006). The protective effects of EPO may be mediated by multiple signaling pathways, such as JAK/STAT, PI3/ Akt, MAPKs, and eNOS (Smith et al. 2003; Cai and Semenza 2004; Rafiee et al. 2005; Burger et al. 2006; Miyata et al. 2006). In the present study, in which the effects of EPO on pressure-overloaded hearts were investigated, Western blotting analysis revealed that the phosphorylation of STAT3 in LV myocardium was significantly accelerated by the treatment with EPO for 8 weeks. Jacoby et al. reported that cardiomyocyte-restricted knockout of STAT3 resulted in higher sensitivity to cardiac fibrosis with advanced age and cardiomyocyte apoptosis in response to lipopolysaccharide (Jacoby et al. 2003). Thus, the accelerated phosphorylation of STAT3 in TAC-EPO observed in the present study may explain, at least in part, the reduced myocardial interstitial fibrosis and cardiomyocyte apoptosis as compared with TAC-PBS. The expression level of phosphorylated Akt was significantly increased in TAC-EPO as compared with TAC-PBS. Akt plays crucial roles in promoting physiological cardiac hypertrophy induced by exercise and in antagonizing pathological hypertrophy elicited by TAC-induced LV pressure overload (DeBosch et al. 2006). It is also possible that the activated Akt contributed to preserved LV function of TAC-EPO in the present study. We also demonstrated that phosphorylation of p38 was significantly depressed in TAC-EPO as compared with TAC-PBS. p38 is located at the downstream pathways of the stimuli from TNF- $\alpha$ and myocardial ischemia, mediating intracellular signaling of myocardial matrix remodeling and impaired cardiac contractility (Kerkela and Force 2006). In addition, inhibition of p38 activity in cultured cardiomyocytes or intact hearts reduces apoptotic cell death in response to various cardiac stresses such as pressure overload and ischemia/reperfusion (Baines and Molkentin 2005; Kerkela and Force 2006). Thus, it is possible that the attenuated phosphorylation of p38 in LV myocardium after the treatment with EPO is involved in the mechanisms for the 
ameliorated myocardial interstitial fibrosis and cardiomyocyte apoptosis, and improved LV function in TACEPO. However, a further study is required to determine the significance of the attenuated phosphorylation of $\mathrm{p} 38$ in the TAC-EPO as compared with TAC-PBS as Nishida et al. (2004) reported that cardiac-specific p38 $\alpha$ knockout mice develop accelerated LV remodeling and myocardial apoptosis in response to TAC as compared with wild-type mice with TAC, and our previous study also demonstrated that one-week TAC in mice deficient in EPO receptors exclusively in nonhematopoietic cells resulted in deteriorated LV remodeling and premature death, which was associated with the depressed phosphorylation of $\mathrm{p} 38$ at 5 hours after TAC (Asaumi et al. 2007).

The expression level of phosphorylated eNOS in LV myocardium was also increased in TAC-EPO as compared with TAC-PBS in the present study. Ruetten et al. (2005) demonstrated that chronic LV pressure overload induced by 6-week abdominal aortic banding in eNOS knockout mice resulted in concentric LV hypertrophy with remarkable myocardial interstitial fibrosis but without LV dysfunction as compared with wild-type mice with LV pressure overload. Furthermore, Burger et al. (2006) reported that antiapoptotic effects of EPO were significantly decreased in murine cardiomyocytes treated with L-NAME, which inhibits NOS activity. Thus, it is conceivable that the increase in phosphorylated eNOS in LV myocardium of TAC-EPO as compared with TAC-PBS is involved, at least in part, in the reduced myocardial interstitial fibrosis and cardiomyocyte apoptosis after TAC. As Akt activates eNOS by phosphorylation of its specific site (Shiojima and Walsh 2002; Boo and Jo 2003), the increased level of phosphorylated Akt induced by EPO receptor-mediated signaling may be responsible for the increased level of phosphorylated eNOS. On the other hand, shear stress stimulates eNOS phosphorylation in both Akt-dependent and -independent manners (Boo and Jo 2003). In the present study, hematocrit level was $69 \%$ in TAC-EPO whereas that in TAC-PBS was $46 \%$. As elevated hematocrit increases blood viscosity (Cinar et al. 1999), which is one of the important determinants of shear stress (Melkumyants et al. 1989), endothelial cells in LV myocardium in TAC-EPO should have been exposed to higher shear stress than those in TAC-PBS. Thus, the activation of eNOS in LV myocardium in TAC-EPO may be explained by both EPO receptormediated Akt activation and increased shear stress induced by erythropoietic action of EPO.

\section{Study limitations}

Several limitations should be mentioned for the present study. Firstly, hematocrit level at 8 weeks after the treatment with EPO was as high as $69 \%$, which is much higher than that observed in patients with chronic kidney disease and anemia treated with EPO. Indeed, death during echocardiography under anesthesia was observed only in TAC-EPO in the present study, and could possibly be explained by a synergistic effect of negative inotropic action of anesthetics and increased LV afterload due to high blood viscosity (Cinar et al. 1999). With regard to patients with chronic kidney disease and anemia, Singh et al. (2006) demonstrated that a target hemoglobin level of $13.5 \mathrm{~g} / \mathrm{dl}$ achieved by treatment with a higher dose of EPO was associated with increased risk of a composite of death, myocardial infarction, hospitalization for heart failure, and stroke as compared with a target hemoglobin level of 11.3 $\mathrm{g} / \mathrm{dl}$. Although not all the patients enrolled in this clinical trial had definite heart diseases, we cannot simply extrapolate the results of the present animal study to clinical application of EPO for patients with LV pressure overload. Secondly, we analyzed only animals that survived for 8 weeks after operation in histological and protein expression analyses. We cannot exclude the possibility that the results might have been different if we had been able to include those animals that died before the end of the experimental protocols. Thirdly, we were unable to differentiate the effects of recombinant human EPO mediated by EPO receptors in the cardiovascular system from those elicited by hematopoietic action of EPO, such as increased shear stress that may activate endothelial eNOS in LV myocardium and possibly increased capability of oxygen delivery to hypertrophied cardiomyocytes that might be exposed to hypoxia (Oka and Komuro 2008). Further studies using mice deficient in EPO receptors exclusively in nonhematopoietic cells are required to address this important issue.

\section{Conclusions}

We were able to demonstrate that recombinant human EPO exerts cardioprotective effects against the development of cardiac remodeling and premature death induced by chronic LV pressure overload by mechanisms independent of coronary angiogenesis. Up-regulation in the phosphorylation of STAT3, Akt, and eNOS and down-regulation in that of p38 may be involved in the cardioprotective effects of EPO.

\section{Acknowledgments}

We thank Ms. Naomi Yamaki, Ms. Fumie Natsui and Ms. Fumie Tatebayashi for their excellent technical assistance. This study was supported in part by Grants-in-Aid for Science Research (Nos. 16209027, 20117009, 22590800) from the Japanese Ministry of Education, Culture, Sports, Science and Technology, Tokyo, Japan

\section{Conflict of Interest}

The authors have no conflict of interest regarding this work.

\section{References}

Asaumi, Y., Kagaya, Y., Takeda, M., Yamaguchi, N., Tada, H., Ito, K., Ohta, J., Shiroto, T., Shirato, K., Minegishi, N. \& Shimokawa, H. (2007) Protective role of endogenous erythropoietin system in nonhematopoietic cells against pressure overload-induced left ventricular dysfunction in mice. Circu- 
lation, 115, 2022-2032.

Baines, C.P. \& Molkentin, J.D. (2005) STRESS signaling pathways that modulate cardiac myocyte apoptosis. J. Mol. Cell. Cardiol., 38, 47-62.

Boo, Y.C. \& Jo, H. (2003) Flow-dependent regulation of endothelial nitric oxide synthase: role of protein kinases. $\mathrm{Am}$. J. Physiol. Cell. Physiol., 285, C499-C508.

Burger, D., Lei, M., Geoghegan-Morphet, N., Lu, X., Xenocostas, A. \& Feng, Q. (2006) Erythropoietin protects cardiomyocytes from apoptosis via up-regulation of endothelial nitric oxide synthase. Cardiovasc. Res., 72, 51-59.

Cai, Z. \& Semenza, G.L. (2004) Phosphatidylinositol-3-kinase signaling is required for erythropoietin-mediated acute protection against myocardial ischemia/reperfusion injury. Circulation, 109, 2050-2053.

Calvillo, L., Latini, R., Kajstura, J., Leri, A., Anversa, P., Ghezzi, P., Salio, M., Cerami, A. \& Brines, M. (2003) Recombinant human erythropoietin protects the myocardium from ischemiareperfusion injury and promotes beneficial remodeling. Proc. Natl. Acad. Sci. USA, 100, 4802-4806.

Cinar, Y., Demir, G., Pac, M. \& Cinar, A.B. (1999) Effect of hematocrit on blood pressure via hyperviscosity. Am. J. Hypertens., 12, 739-743.

DeBosch, B., Treskov, I., Lupu, T.S., Weinheimer, C., Kovacs, A., Courtois, M. \& Muslin, A.J. (2006) Akt1 is required for physiological cardiac growth. Circulation, 113, 2097-2104.

Digicaylioglu, M., Bichet, S., Marti, H.H., Wenger, R.H., Rivas, L.A., Bauer, C. \& Gassmann, M. (1995) Localization of specific erythropoietin binding sites in defined areas of the mouse brain. Proc. Natl. Acad. Sci. USA, 92, 3717-3720.

Fukui, S., Fukumoto, Y., Suzuki, J., Saji, K., Nawata, J., Shinozaki, T., Kagaya, Y., Watanabe, J. \& Shimokawa, H. (2009) Diabetes mellitus accelerates left ventricular diastolic dysfunction through activation of the renin-angiotensin system in hypertensive rats. Hypertens. Res., 32, 472-480.

Grimm, C., Wenzel, A., Groszer, M., Mayser, H., Seeliger, M., Samardzija, M., Bauer, C., Gassmann, M. \& Remé, C.E. (2002) HIF-1-induced erythropoietin in the hypoxic retina protects against light-induced retinal degeneration. Nat. Med., 8, 718-724.

Harada, K., Komuro, I., Shiojima, I., Hayashi, D., Kudoh, S., Mizuno, T., Kijima, K., Matsubara, H., Sugaya, T., Murakami, K. \& Yazaki, Y. (1998) Pressure overload induces cardiac hypertrophy in angiotensin II type 1A receptor knockout mice. Circulation, 97, 1952-1959.

Hunt, S.A. (2005) American College of Cardiology; American Heart Association Task Force on Practice Guidelines (Writing Committee to Update the 2001 Guidelines for the Evaluation and Management of Heart Failure). ACC/AHA 2005 guideline update for the diagnosis and management of chronic heart failure in the adult: a report of the American College of Cardiology/American Heart Association Task Force on Practice Guidelines (Writing Committee to Update the 2001 Guidelines for the Evaluation and Management of Heart Failure). J. Am. Coll. Cardiol., 46, e1-e82.

Iwanaga, Y., Kihara, Y., Hasegawa, K., Inagaki, K., Yoneda, T., Kaburagi, S., Araki, M. \& Sasayama, S. (1998) Cardiac endothelin-1 plays a critical role in the functional deterioration of left ventricles during the transition from compensatory hypertrophy to congestive heart failure in salt-sensitive hypertensive rats. Circulation, 98, 2065-2073.

Izumiya, Y., Shiojima, I., Sato, K., Sawyer, D.B., Colucci, W.S. \& Walsh, K. (2006) Vascular endothelial growth factor blockade promotes the transition from compensatory cardiac hypertrophy to failure in response to pressure overload. Hypertension, 47, 887-893.

Jacoby, J.J., Kalinowski, A., Liu, M.G., Zhang, S.S., Gao, Q., Chai, G.X., Ji, L., Iwamoto, Y., Li, E., Schneider, M., Russell, K.S. \& Fu, X.Y. (2003) Cardiomyocyte-restricted knockout of
Stat3 results in higher sensitivity to inflammation, cardiac fibrosis, and heart failure with advanced age. Proc. Natl. Acad. Sci. USA, 100, 12929-12934.

Jessup, M. \& Brozena, S. (2003) Heart failure. N. Engl. J. Med., 348, 2007-2018.

Kerkela, R. \& Force, T. (2006) p38 mitogen-activated protein kinase: a future target for heart failure therapy? J. Am. Coll. Cardiol., 48, 556-558.

Koury, M.J. \& Bondurant, M. (1990) Erythropoietin retards DNA breakdown and prevents programmed death in erythroid progenitor cells. Science, 248, 378-381.

Li, L., Takemura, G., Li, Y., Miyata, S., Esaki, M., Okada, H., Kanamori, H., Khai, N.C., Maruyama, R., Ogino, A., Minatoguchi, S., Fujiwara, T. \& Fujiwara, H. (2006) Preventive effect of erythropoietin on cardiac dysfunction in doxorubicin-induced cardiomyopathy. Circulation, 113, 535-543.

McMurray, J.J. \& Pfeffer, M.A. (2005) Heart failure. Lancet, 365, $1877-1889$.

Melkumyants, A.M., Balashov, S.A. \& Khayutin, V.M. (1989) Endothelium dependent control of arterial diameter by blood viscosity. Cardiovasc. Res., 23, 741-747.

Miyata, S., Maruyama, R., Li, L., Higuchi, M., Minatoguchi, S., Fujiwara, T. \& Fujiwara, H. (2006) Reduction of inflammatory cytokine expression and oxidative damage by erythropoietin in chronic heart failure. Cardiovasc. Res., 71, 684-694.

Nakano, M., Satoh, K., Fukumoto, Y., Ito, Y., Kagaya, Y., Ishii, N., Sugamura, K. \& Shimokawa, H. (2008) Important role of erythropoietin receptor to promote VEGF expression and angiogenesis in peripheral ischemia in mice. Circ. Res., 100, 662-669.

Namiuchi, S., Kagaya, Y., Ohta, J., Shiba, N., Sugi, M., Oikawa, M., Kunii, H., Yamao, H., Komatsu, N., Yui, M., Tada, H., Sakuma, M., Watanabe, J., Ichihara, T. \& Shirato, K. (2005) High serum erythropoietin level is associated with smaller infarct size in patients with acute myocardial infarction who undergo successful primary percutaneous coronary intervention. J. Am. Coll. Cardiol., 45, 1406-1412.

Nian, M., Lee, P., Khaper, N. \& Liu, P. (2004) Inflammatory cytokines and postmyocardial infarction remodeling. Circ. Res., 94, 1543-1553.

Nishida, K., Yamaguchi, O., Hirotani, S., Hikoso, S., Higuchi, Y., Watanabe, T., Takeda, T., Osuka, S., Morita, T., Kondoh, G., Uno, Y., Kashiwase, K., Taniike, M., Nakai, A., Matsumura, Y., Miyazaki, J., Sudo, T., Hongo, K., Kusakari, Y., Kurihara, S., Chien, K.R., Takeda, J., Hori, M. \& Otsu, K. (2004) p38 alpha mitogen-activated protein kinase plays a critical role in cardiomyocyte survival but not in cardiac hypertrophic growth in response to pressure overload. Mol. Cell. Biol., 24, 1061110620.

Nishiya, D., Omura, T., Shimada, K., Matsumoto, R., Kusuyama, T., Enomoto, S., Iwao, H., Takeuchi, K., Yoshikawa, J. \& Yoshiyama, M. (2006) Effects of erythropoietin on cardiac remodeling after myocardial infarction. J. Pharmacol. Sci., 101, 31-39.

Oka, T. \& Komuro, I. (2008) Molecular mechanisms underlying the transition of cardiac hypertrophy to heart failure. Circ. J., 72, A13-A16.

Panidis, I.P., Kotler, M.N., Ren, J.F., Mintz, G.S., Ross, J. \& Kalman, P. (1984) Development and regression of left ventricular hypertrophy. J. Am. Coll. Cardiol., 3, 1309-1320.

Parsa, C.J., Matsumoto, A., Kim, J., Riel, R.U., Pascal, L.S., Walton, G.B., Thompson, R.B., Petrofski, J.A., Annex, B.H., Stamler, J.S. \& Koch, W.J. (2003) A novel protective effect of erythropoietin in the infarcted heart. J. Clin. Invest., 112 999-1007.

Rafiee, P., Shi, Y., Su, J., Pritchard, K.A. Jr., Tweddell, J.S. \& Baker, J.E. (2005) Erythropoietin protects the infant heart against ischemia-reperfusion injury by triggering multiple 
signaling pathways. Basic Res. Cardiol., 100, 187-197.

Ruetten, H., Dimmeler, S., Gehring, D., Ihling, C. \& Zeiher, A.M. (2005) Concentric left ventricular remodeling in endothelial nitric oxide synthase knockout mice by chronic pressure overload. Cardiovasc. Res., 66, 444-453.

Rui, T., Feng, Q., Lei, M., Peng, T., Zhang, J., Xu, M., Abel, E.D., Xenocostas, A. \& Kvietys, P.R. (2005) Erythropoietin prevents the acute myocardial inflammatory response induced by ischemia/reperfusion via induction of AP-1. Cardiovasc. Res., 65, 719-727.

Satoh, K., Kagaya, Y., Nakano, M., Ito, Y., Ohta, J., Tada, H., Karibe, A., Minegishi, N., Suzuki, N., Yamamoto, M., Ono, M., Watanabe, J., Shirato, K., Ishii, N., Sugamura, K. \& Shimokawa, H. (2006) Important role of endogenous erythropoietin system in recruitment of endothelial progenitor cells in hypoxia-induced pulmonary hypertension in mice. Circulation, 113, 1442-1450.

Schultz, J.E., Witt, S.A., Nieman, M.L., Reiser, P.J., Engle, S.J., Zhou, M., Pawlowski, S.A., Lorenz, J.N., Kimball, T.R. \& Doetschman, T. (1999) Fibroblast growth factor-2 mediates pressure-induced hypertrophic response. J. Clin. Invest., 104, 709-719.

Schultz, J.J., Witt, S.A., Glascock, B.J., Nieman, M.L., Reiser, P.J., Nix, S.L., Kimball, T.R. \& Doetschman, T. (2002) TGF-beta1 mediates the hypertrophic cardiomyocyte growth induced by angiotensin II. J. Clin. Invest., 109, 787-796.

Shiojima, I. \& Walsh, K. (2002) Role of Akt signaling in vascular homeostasis and angiogenesis. Circ. Res., 90, 1243-1250.

Shoemaker, C.B. \& Mitsock, L.D. (1986) Murine erythropoietin gene: cloning, expression, and human gene homology. Mol. Cell. Biol., 6, 849-858.

Singh, A.K., Szczech, L., Tang, K.L., Barnhart, H., Sapp, S.,
Wolfson, M., Reddan, D. \& CHOIR Investigators. (2006) Correction of anemia with epoetin alfa in chronic kidney disease. N. Engl. J. Med., 355, 2085-2098.

Smith, K.J., Bleyer, A.J., Little, W.C. \& Sane, D.C. (2003) The cardiovascular effects of erythropoietin. Cardiovasc. Res., 59, 538-548.

Tada, H., Kagaya, Y., Takeda, M., Ohta, J., Asaumi, Y., Satoh, K., Ito, K., Karibe, A., Shirato, K., Minegishi, N. \& Shimokawa, H. (2006) Endogenous erythropoietin system in non hematopoietic lineage cells plays a protective role in myocardial ischemia/reperfusion. Cardiovasc. Res., 71, 466-477.

Ueda, K., Takano, H., Niitsuma, Y., Hasegawa, H., Uchiyama, R., Oka, T., Miyazaki, M., Nakaya, H. \& Komuro, I. (2010) Sonic hedgehog is a critical mediator of erythropoietininduced cardiac protection in mice. J. Clin. Invest., 120, 2016-2029.

Wen, D., Boissel, J.P., Tracy, T.E., Gruninger, R.H., Mulcahy, L.S., Czelusniak, L., Goodman, M. \& Bunn, H.F. (1993) Erythropoietin structure-function relationships: high degree of sequence homology among mammals. Blood, 82, 1507-1516.

Wright, G.L., Hanlon, P., Amin, K., Steenbergen, C., Murphy, E. \& Arcasoy, M.O. (2004) Erythropoietin receptor expression in adult rat cardiomyocytes is associated with an acute cardioprotective effect for recombinant erythropoietin during ischemia- reperfusion injury. FASEB J., 18, 1031-1033.

van der Meer, P., Lipsic, E., Henning, R.H., Boddeus, K., van der Velden, J., Voors, A.A., van Veldhuisen, D.J., van Gilst, W.H. \& Schoemaker, R.G. (2005) Erythropoietin induces neovascularization and improves cardiac function in rats with heart failure after myocardial infarction. J. Am. Coll. Cardiol., 46, 125-133. 